\section{Adherencia al tratamiento en las enfermedades crónicas y la vivencia del paciente}

\section{Adherence to treatment in chronic diseases and the patient's experience}

\section{Sr. Editor:}

Suficiente ha sido escrito acerca de las negativas consecuencias clínicas y económicas ligadas a la no adherencia al tratamiento médico. El problema adquiere mayor peso en las enfermedades crónicas, en las que se espera un activo rol del paciente como agente de su propio cuidado.

Más de 100.000 trabajos han sido publicados sobre el tema y más de 200 factores han sido relacionados con la no adherencia al tratamiento. Los resultados de esta tarea no han sido consistentes y hasta hoy no existe acuerdo respecto de los factores predictivos de la adherencia al tratamiento.

Una pregunta central en el estudio de la no adherencia al tratamiento es por qué una persona elige -consciente o inconscientemente- no seguir el consejo médico, a pesar de conocer las consecuencias negativas de esta decisión. Es evidente que una mirada puramente cognitiva no explica completamente el problema. De hecho y más frecuentemente que lo esperado, las personas se comportan de manera "irracional", una conducta cuya comprensión requeriría incorporar variables diferentes y complementarias a las cognitivas, p.ej. las teorías subjetivas del paciente respecto de su enfermedad o el significado que atribuye al tratamiento.

Hasta la fecha, buena parte de las teorías explicativas de la adherencia la han planteado más como una conducta que como parte de un proceso psicológico. De hecho, gran parte de las teorías psicológicas explicativas (teoría de la acción razonada, el modelo de creencias en salud, modelo transteórico, entre otros), se han basado en la premisa que las personas toman decisiones racionales y objetivas respecto de su tratamiento, después de sopesar las ventajas y desventajas de ello. Ajzen ha planteado que el logro de una conducta, p.ej. la adherencia, dependería fuertemente de la motivación (intención), la percepción de control que se tiene sobre ella y las normas subjetivas sociales ${ }^{2}$.

Estas conceptualizaciones permiten predecir en cierta medida la no-adherencia intencional, i.e. aquel proceso mediante el cual el paciente activamente decide no cooperar ni ser agente activo en el cuidado de su salud. Su poder predictivo disminuye, sin embargo, cuando se trata de evaluar la no-adherencia no-intencional, i.e. baja adherencia al tratamiento como una expresión de deficiencias del paciente durante el proceso de tratamiento, como olvidos, fallas en la planificación etc. ${ }^{3}$.

Inicialmente se pensó que la baja adherencia no intencional estaba ligada a factores demográficos, como la baja escolaridad, edad, etc. Datos recientes, sin embargo, sugieren que en ambos tipos de adherencia, las creencias, actitudes y temores respecto del tratamiento juegan un papel importante ${ }^{4}$. Entre ellas figuran la percepción de necesidad del medicamento y la percepción de su accesibilidad.

En este contexto, dos temas adquieren relevancia. Por una parte, considerar ambos tipos de no adherencia al momento de diseñar estrategias focalizadas. Por otra parte, la necesidad de incluir la experiencia del paciente en cualquier modelo comprensivo del problema. Es innecesario enfatizar que esta comprensión debiera incluir no sólo los factores cognitivos de esta experiencia, sino además la vivencia del paciente en relación a su enfermedad, en sus aspectos conscientes e inconscientes. En términos de Kleinman, el padecimiento (illness) del paciente ${ }^{5}$.

Esta experiencia del hombre con su enfermedad y su padecer, propone un escenario donde se despliegan necesidades y conflictos tempranos, que inevitablemente se reviven en la relación médico/paciente. Así p.ej., una capacidad necesaria en cualquier tratamiento, como es el poder confiar en otro y permitir ser ayudado, se facilita cuando se ha tenido una experiencia temprana de cuidado y apego seguro. Lo mismo puede postularse respecto del temor a la dependencia -psíquica, biológica- con que el paciente y eventualmente su médico, configuran la relación del tratamiento. El fracaso en la elaboración de este tema podría traducirse en una baja adherencia, comprensible desde el temor a depender del medicamento y del médico tratante ${ }^{6}$.

El escenario descrito involucra tanto al paciente como a su médico tratante, exigiendo de alguna manera una resolución delos conflictos descritos. La elaboración exitosa de este proceso promueve la alianza terapéutica y la consecuente mejoría 
en la adherencia. En breve, volver a las raíces del quehacer médico, considerando al paciente como el centro de nuestra actividad.

\section{Luis Alvarado ${ }^{1,2}$}

${ }^{1}$ Departamento de Psiquiatría y Salud Mental

Oriente. Facultad de Medicina, Universidad de Chile. Santiago de Chile. ${ }^{2}$ Departamento de Psicología. Facultad de Ciencias Sociales, Universidad de Chile. Santiago de Chile.

\section{Referencias}

1. Vermeire E, Hearnshaw M, Van Royen P, Denekens J. Patient adherence to treatment: three decades of research. A comprehensive review. J Clin Pharm Ther 2001; 26: 331-42.

2. Ajzen I. The theory of planned behavior. Organ Behav Hum Dec 1991; 50: 179-211.

3. Lehane E, McCarthy G. An examination of the intentional and unintentional aspects of medication non-adherence in patients diagnosed with hypertension. J Clin Nurs 2007; 16: 698-706.
4. Gadkari AS, McHorney CA. Unintentional non-adherence to chronic prescription medications: How unintentional is it really? BMC Health Services Research 2012; 12: 98. Disponible en: http://www.biomedcentral. com/1472-6963/12/98 (Consultado el 15 de octubre de 2015).

5. Kleinman A. The Illness narratives. Suffering, Healing \& the Human Condition (1998). Basic Books, Perseus Basics Books, 1-28.

6. Alfonso C. Dynamic psychopharmacology and treatment adherence. J Amer Acad Psychoanal 2009; 37: 26985. Disponible en http://www.pep-web.org/document. php?id=jaa.037.0269a (Consultado el 8 de agosto de 2015).

Conflictos de intereses: ninguno que declarar.

Correspondencia a:

Dr. Luis Alvarado

lalvarado@uchile.cl 